\title{
Investigating the interaction of EtpA and flagellin from enterotoxigenic Escherichia coli (ETEC)
}

\author{
Clifford M Ntui ${ }^{\mathrm{a}}$ and Wolf-Dieter Schubert ${ }^{\mathrm{b}}$ \\ Structural Biology of Infectious Diseases, Department of Biochemistry, University of Pretoria. \\ a Department of Biochemistry, University of Pretoria, Pretoria 0003, South Africa, \\ ntuimanyo@gmail.com \\ ${ }^{b}$ Department of Biochemistry, University of Pretoria, Pretoria 0003, South Africa, wolf- \\ dieter.schubert@up.ac.za
}

\section{Abstract}

Enterotoxigenic Escherichia coli (ETEC) is the most common diarrhoea-inducing bacterium with high mortality rates especially in young children. As part of its infection strategy, ETEC invades and colonizes the epithelium of the small intestine where it secretes heat-labile and/or heat-stable enterotoxins to induce diarrhoea. Pathogenicity is further controlled by plasmid and chromosome encoded virulence factors. One of these, EtpA, is a $170 \mathrm{kDa}$ glycoprotein secreted by a two-partner secretion system. EtpA is essential for the effective colonization of intestinal epithelia. As an adhesin it links flagellin (FliC) at the tip of ETEC flagellae to the host cell surface. Previous studies have demonstrated that the Nterminus of EtpA is involved in interactions with flagellin. However, structural information on EtpA and its interaction with flagellin is currently unavailable. Here we describe the first crystal structure of EtpA $^{69-477}$ from ETEC and confirm its interaction with FliC also from ETEC. Structurally EtpA ${ }^{69-477}$ forms an extended $\beta$-helix known to be particularly stable and hence useful for secreted proteins. Our molecular pull-down assay indicated that Etp $A^{69-477}$ binds to ETEC FliC in a 1:1 ratio. By contrast, it does not bind to FliC from E. coli ATCC 8739. These data provide insight into the structure of ETEC EtpA ${ }^{69-477}$ and its interaction with ETEC FliC with possible implications for vaccine design.

\section{References}

Enguo F, Silke F, Francoise J-D, and Matthias M (2012) Two-partner secretion of gram-negative bacteria. A single-barrel protein enables transport across the outer membrane. Journal of Biological Chemistry 287(4), 2591-2599.

Fleckenstein J, Sheikh A, and Qadri F (2014) Novel antigens for enterotoxigenic Escherichia coli vaccines. Expert Rev. Vaccines 13, 631-639. 